\title{
Lithium Indium Diselenide: A New Scintillator for Neutron Imaging
}

Eric Lukosi ${ }^{1, *}$, Elan Herrera ${ }^{1}$, Daniel Hamm ${ }^{1}$, Kyung-Min Lee $^{1}$, Brenden Wiggins ${ }^{2}$, Pavel Trtik ${ }^{3}$, Dayakar Penumadu ${ }^{1}$, Stephen Young ${ }^{1}$, Louis Santodonato ${ }^{4}$, Hassina Bilheux ${ }^{4}$, Arnold Burger ${ }^{5}$, Liviu Matei ${ }^{5}$, Ashley C. Stowe ${ }^{1,2}$

${ }^{1}$ University of Tennessee, Knoxville, TN, USA

${ }^{2}$ Y-12 National Security Complex, Oak Ridge, TN, USA

${ }^{3}$ Paul Scherrer Institut, Villigen, CH-5232, Switzerland

${ }^{4}$ Oak Ridge National Laboratory, Oak Ridge, TN, USA

${ }^{5}$ Fisk University, Nashville, TN, USA

\begin{abstract}
Lithium indium diselenide, ${ }^{6} \mathrm{LiInSe}_{2}$ or LISe, is a newly developed neutron detection material that shows both semiconducting and scintillating properties. This paper reports on the performance of scintillating LISe crystals for its potential use as a converter screen for cold neutron imaging. The spatial resolution of LISe, determined using a 10\% threshold of the Modulation Transfer Function (MTF), was found to not scale linearly with thickness. Crystals having a thickness of $450 \mu \mathrm{m}$ or larger resulted in an average spatial resolution of $67 \mu \mathrm{m}$, and the thinner crystals exhibited an increase in spatial resolution down to the Nyquist frequency of the CCD. The highest measured spatial resolution of $198 \mu \mathrm{m}$ thick LISe $(27 \mu \mathrm{m})$ outperforms a commercial $50 \mu \mathrm{m}$ thick $\mathrm{ZnS}(\mathrm{Cu}):{ }^{6} \mathrm{LiF}$ scintillation screen by more than a factor of three. For the LISe dimensions considered in this study, it was found that the light yield of LISe did not scale with its thickness. However, absorption measurements indicate that the ${ }^{6} \mathrm{Li}$ concentration is uniform and the neutron absorption efficiency of LISe as a function of thickness follows general nuclear theory. This suggests that the differences in apparent brightness observed for the LISe samples investigated may be due to a combination of secondary charged particle escape, scintillation light transport in the bulk and across the LISe-air interface, and variations in the activation of the scintillation mechanism. Finally, it was found that the presence of ${ }^{115} \mathrm{In}$ and its long-lived ${ }^{116} \mathrm{In}$ activation product did not result in ghosting (memory of past neutron exposure), demonstrating potential of LISe for imaging transient systems.
\end{abstract}

Keywords: $\quad$ Neutron imaging; Neutron detection; Scintillator; Lithium indium diselenide; LISe; Cold neutrons

\section{Introduction}

The interactions of cold neutrons with matter is a function of nuclear cross sections and crystalline structure [1], enabling neutrons to probe matter in a variety of ways. One such way is cold neutron imaging, where generated radiographs (or 3D images) reveal information about the interior or structural properties of imaged materials and devices [2]. Many modern thermal/cold neutron

${ }^{*}$ Corresponding author: +18659746568

Fax: +1 8659740668

Email address: elukosi@utk.edu (Eric Lukosi) 
imaging systems utilize a scintillation screen doped with a neutron-sensitive isotope (e.g., ${ }^{6} \mathrm{Li},{ }^{10} \mathrm{~B}$, $\left.{ }^{\text {nat }} \mathrm{Gd}\right)$ coupled to a light collection apparatus. The neutron interaction results in secondary charged particles that result in light production via scintillation. The range of secondary particles and thickness of the scintillator are critical factors in the spatial resolution of the imaging system. The range of secondary particles defines the intrinsic point spread function of the converter, and the thickness of the converter/scintillation screen results in a spread of the scintillation photons during internal transport to the surface. The result is overlapping point spread functions at the digitizer plane (e.g., CCD camera) generated from adjacent points, thereby blurring the image [3]. Therefore, the ideal neutron converter yields a narrow intrinsic point spread function (range of secondary charged particles), a large detection efficiency per unit path length, and generates a large number of light photons per neutron interaction. These characteristics limit both image blurring from thick scintillators and exposure time to gain the necessary signal-to-noise ratio (SNR) [4].

A general rule of thumb for scintillator-based converters assumes that the achievable spatial resolution is limited to the thickness of the scintillation screen, such as $\mathrm{ZnS}:{ }^{6} \mathrm{LiF}$ scintillation screens. Gadolinium-based scintillation screens are currently being explored for high resolution imaging because of its unparalleled neutron cross section and low energy of the conversion electrons. However, the gamma-rays produced result in image degradation and errors associated with false neutron counting [5]. Still, efforts are underway to increase the spatial resolution using Gadolinium-based scintillations screens by using micron-sized, ${ }^{157} \mathrm{Gd}$ enriched, scintillation screens [6]. Preliminary results have demonstrated a resolution of $7.6 \mu \mathrm{m}$ [7] and recent results have yielded a resolution of $5.4 \mu \mathrm{m}$ [8]. Another group has utilized infinity-corrected optics and an alternative Gadolinium-containing scintillation screen $\left(\mathrm{Gd}_{3} \mathrm{Ga}_{5} \mathrm{O}_{12}\right.$ : $\left.\mathrm{Eu}^{3+}\right)$, called GGG, and demonstrated a spatial resolution of $14.8 \mu \mathrm{m}$ [9]. It should also be noted that GGG is optically transparent, and their experiment was conducted with a $1 \mathrm{~mm}$ thick scintillator. This result indicates that the resolution achieved was much higher than the thickness of scintillator, which is not possible on this scale using polycrystalline or epoxied scintillators, such as $\mathrm{ZnS}:{ }^{6} \mathrm{LiF}$ scintillation screens.

In an effort to identify additional converter screens that do not exhibit the dependence of spatial resolution on thickness while also maintaining all other desired attributes for neutron imaging, a new scintillating material, lithium indium diselenide (LISe), was investigated in this study [10]. Enriched in ${ }^{6} \mathrm{Li}$ to 95 at\%, LISe provides the needed absorption efficiency and is optically transparent, demonstrating potential for overcoming limitations with existing neutron imaging converter materials. This paper reports on the efficacy of LISe for neutron imaging, considering light yield, neutron absorption efficiency, and spatial resolution as a function of scintillator thickness and surface finish.

\section{Lithium indium diselenide}

Lithium indium diselenide, or LISe, is a I-III-VI $\mathrm{I}_{2}$ chalcopyrite with neutron detection capability via the ${ }^{6} \mathrm{Li}(\mathrm{n}, \mathrm{t})^{4} \mathrm{He}$ reaction with a large Q-value of $4.78 \mathrm{MeV}$. The light yield of LISe has been determined to be 9,000 photons/MeV with a peak wavelength emission at $512 \mathrm{~nm}$ and a principle decay time constant of $31 \pm 1 \mathrm{~ns}$ [11]. The mechanism of scintillation has yet to be identified, but appears to be reproducible. The 24 at $\%{ }^{6} \mathrm{Li}$ concentration in LISe yields a mean free path for thermal neutrons of $920 \mu \mathrm{m}$, but the presence of ${ }^{115} \mathrm{In}$ reduces the ${ }^{6} \mathrm{Li}$-based reactions to an effective absorption efficiency of $82 \%$ at thermal energy. The thermal neutron mean free path of LISe is smaller than any other lithium-containing solid-state scintillator known to the authors, and the high cold neutron absorption efficiency holds promise for its application to neutron imaging. 
However, the decay time constant of ${ }^{116}$ In and the associated ionizing radiation emitted may limit the efficacy of LISe in dynamic neutron imaging studies, and is evaluated in this paper.

\section{Experimental Procedure}

\subsection{Neutron Absorption Efficiency}

Experiments were conducted at the Oak Ridge National Laboratory (ORNL) High Flux Isotope Reactor (HFIR) CG-1D neutron imaging test station and beam line [12] and at the beam line for Neutron Optics and other Approaches (BOA) at Paul Scherrer Institut (PSI) [13]. Neutron absorption, related to detection efficiency, for each LISe sample was determined by evaluating the attenuation through LISe using a $50 \mu \mathrm{m} \mathrm{ZnS(Cu):}{ }^{6} \mathrm{LiF}$ scintillation screen. After open beam and dark field image corrections, the measured absorption, Iabs, is converted into absorption efficiency, $\varepsilon$, using equation 1 , where $I_{o}$ is the measured intensity at a given location from corrected open beam image, $\mathrm{x}$ is the thickness of the absorber/detector, and $\lambda$ is the neutron mean free path.

$$
\varepsilon=\frac{I_{o}-I_{a b s}}{I_{o}}=1-e^{-x / \lambda}
$$

\subsection{LISe Relative Light Yield (RLY)}

The scintillation homogeneity and intensity for each sample was investigated by mounting the LISe samples onto a thin aluminum support plate and placing them in the position of the $\mathrm{ZnS}(\mathrm{Cu}):{ }^{6} \mathrm{LiF}$ scintillation screen. At each beam line, the available optical transfer and digitation systems were utilized. At HFIR, two optical systems were used. The first utilizes lenses and a 45 degree mirror to focus onto a CCD camera (DW 936N-BV iKon-L ANDOR ${ }^{\mathrm{TM}}$ ). The second setup at BOA is similar to the first, but uses an ANDOR ${ }^{\mathrm{TM}}$ Zyla 4.2 sCMOS. Both imaging systems can be used with 25 to $200 \mu \mathrm{m} \mathrm{ZnS(Cu):}{ }^{6} \mathrm{LiF}$ (2:1 ratio) convertor screens (a $50 \mu \mathrm{m}$ thick scintillator was used for measurements reported in this study) deposited on an aluminum support plate with a peak emission at $530 \mathrm{~nm}$ from RC TRITEC. The relative light yield (RLY) measured by each sample was determined by dividing the acquired open beam images with each LISe sample by the response of the $50 \mu \mathrm{m} \mathrm{ZnS}(\mathrm{Cu}):{ }^{6} \mathrm{LiF}$ converter. This serves to both remove the contribution of any variations of the neutron beam intensity and provide a relative measure of its light yield compared to a commonly used neutron imaging scintillator.

\subsection{Spatial Resolution}

The spatial resolution of all LISe samples was determined using the knife-edge technique [14] or visual inspection with the PSI Siemens Star test pattern [15]. For the knife-edge technique, the sharp edge of a neutron absorbing mask in the presence of a uniform neutron field results in a predictable profile response called the edge spread function (ESF). The ESF is the convolution of the line spread function (LSF) and neutron intensity profile across the absorbing edge [14]. For step profiles, differentiation of the ESF results in the LSF. Assuming that the neutron imaging system is shift invariant, the width of the LSF profile describes the width of the system point spread function (PSF), which is a fundamental quantity used to describe the resolution of imaging systems.

The imaging system PSF is equal to the convolution of the various response functions from its components, which includes the beam divergence (geometric unsharpness), scattering degradation (knife-edge unsharpness), scintillator unsharpness, and the finite sampling frequency of the digitizer [14]. Further, the LISe scintillators tested are very thick $(0.2-1 \mathrm{~mm})$ when compared to 
typical $\mathrm{ZnS}(\mathrm{Cu}){ }^{6} \mathrm{LiF}$ screens, but also optically clear, which may lead to a defocusing contribution from the optical transfer between the scintillator and CCD. The gadolinium knife-edges used in these tests are thin-films, and owing to the current technological capabilities of lithographic printing, scattering degradation can be ignored. Therefore, the relevant contributing components of the imaging system are the scintillator unsharpness, beam divergence, and the optical transfer and digitization system (i.e., the lenses and CCD).

For an ideal cold neutron imaging beam line, the beam divergence is a function of the beam aperture diameter and aperture-object and object-scintillator distances. These parameters define the angular distribution of neutrons at the object plane and can be represented as a boxcar function in one dimension. The governing equation for the beam divergence, $\mathrm{h}_{\mathrm{B}}$, in one dimension is [16]

$$
h_{B}(x)=\operatorname{rect}\left(\frac{x^{\prime}-x_{o^{\prime}}}{2 \cdot d \cdot \operatorname{Tan}(\theta)}\right) \text {, }
$$

where $\mathrm{x}_{0}{ }^{`}$ is the location of the knife-edge in the object plane, $\mathrm{x}^{`}$ is the position on the converter plane, $d$ is the distance between the knife-edge and the scintillator, and $\theta$ is the beam divergence. The camera is also described by a boxcar function, where the width is equal to the pixel pitch $(\Delta \mathrm{p})$ of the camera, $\mathrm{x}_{\mathrm{o}}$ is the position on the converter, and $\mathrm{x}$ is the location on the CCD.

$$
h_{C C D}(x)=\operatorname{rect}\left(\frac{x-x_{o}}{\Delta p}\right)
$$

The CCD and the sCMOS cameras used at the CG-1D beam line have pixel pitches of $13.5 \mu \mathrm{m}$ and $6.5 \mu \mathrm{m}$, respectively. The magnification between the scintillator and the CCD chip of each camera is 0.37 and 0.50 , respectively, resulting in an effective pixel size of $36.5 \mu \mathrm{m}$ and $13 \mu \mathrm{m}$ where the radiograph is formed.

For LISe, the scintillator unsharpness is due to a variety of factors. The fundamental component of scintillator unsharpness is the $47 \mu \mathrm{m}$ range of the secondary particles from the ${ }^{6} \mathrm{Li}\left(\mathrm{n},{ }^{3} \mathrm{H}\right){ }^{4} \mathrm{He}$ reaction. These products are emitted isotropically and antiparallel, and the average angle of emission is $45^{\circ}$, such that the average spread parallel to the camera plane is $33 \mu \mathrm{m}$. Furthermore, the thickness of LISe and its optically translucent nature also play a combined role. For thinner samples, the range of the secondary particles will vary the light yield as a function of emission angle due to particle escape, complicating the analysis. For thicker samples, the majority of neutron interactions will deposit the full reaction energy within the scintillator. The light generated will then travel through the birefringent, high index of refraction LISe crystal [17]. The optical transfer system has a fixed focus length and depth of field, both of which were not optimized for the measurements conducted here. Owing to the complexity, the scintillator unsharpness and optical transfer system were considered a single component in the analysis. Equation 4 was found to best fit the measured LSF [14], where $\alpha$ is the resolution parameter, $\mathrm{x}$ is the 1D position on the image plane, and $x_{0}$ is the knife-edge location (object plane position). The resulting measured system response, $\mathrm{L}$, is now described by equation 5 .

$$
L\left(x, x_{o}\right)=\frac{\alpha / \pi}{1+\alpha^{2}\left(x-x_{o}\right)^{2}}
$$

$$
h_{m}(x)=h_{B}(x) * h_{C C D}(x) * L\left(x, x_{o}\right)
$$


For measured knife-edge responses, linear interpolation was used to super sample the CCD response along each row at ten times its sampling frequency. Then, the edge location for each row of image data was determined through a least regression on each differentiated row (i.e., the LSF). Each row was subsequently shifted to correct for the slanted edge and each column of each row were averaged together to minimize the contribution of noise. Then, the super-sampled and averaged LSF was truncated by taking a fixed and equal number of data points on each side of the peak. This latter operation removes the shift variant response resulting from varying edge locations when conducting a Discrete Fourier Transform (DFT) [18]. The absolute value of the DFT is called the Modulation Transfer Function (MTF) and is a measure of the output-to-input system response, thereby defining the loss of contrast at a given spatial frequency and therefore, the system spatial resolution. Equation 6 describes the MTF, where $\mathrm{N}$ is the length of the truncated LSF, $\mathrm{j}$ is the index of the row position, and $\omega$ is the spatial frequency in cycles per $\mathrm{N}$.

$$
\operatorname{MTF}(\omega)=\left|\sum_{j=1}^{N} L S F[[j]] \cdot e^{-2 \pi i(j-1) \frac{(\omega-1)}{N}}\right|
$$

In terms of equation 5, the MTF can be described through equation 7, where A is a normalization constant.

$$
\operatorname{MTF}(d, \theta, \alpha, \omega)=A\left|\operatorname{sinc}(d \cdot \omega \cdot \operatorname{Tan}(\theta)) \operatorname{sinc}(\omega \cdot \Delta p) e^{-i \frac{\omega}{\alpha_{S c}}}\right|
$$

The choice of the input-to-output attenuation to define the spatial resolution is somewhat arbitrary, although a value of $10 \%$ is often used to find the corresponding frequency, $\omega$. The system resolution, $\mathrm{X}_{\mathrm{res}}$, is described through equation 8, where the subscripts DFT and FT refer to equations 6 and 7, respectively. Of note is the factor of two included in equation 8, which converts line pairs to lines, defining the smallest resolvable feature in the image.

$$
x_{r e s, D F T}=\frac{1}{2 \omega} ; \quad x_{r e s, F T}=\frac{\pi}{\omega}
$$

In order to quantify the intrinsic spatial resolution of LISe, its response to the knife-edge test is ideally conducted without influence from the other components of the imaging system. For the beam divergence, this can be accomplished through the knife-edge technique when $d$, the separation between the knife-edge and scintillator is zero. The CCD, however, cannot be removed from the measurement, and therefore, any response measured will be a function of both the scintillator and digitizer. However, if the digitizer passes spatial frequencies much higher than the scintillator, then this term can be safely ignored as it will contribute little to the measured inputto-output attenuation.

\section{Results and Discussion}

A total of seven LISe crystals were measured from three different crystal growth boules. The thickness of each sample ranged from $0.2 \mathrm{~mm}$ to $1 \mathrm{~mm}$ with an active area between 4 and $50 \mathrm{~mm}^{2}$. Two samples were cut and polished into several pieces of differing thickness and surface roughness to provide a comparison of LISe properties as a function of these parameters. A list of each sample, measurement location/camera system, its thickness, and its boule number identifier is provided in Table 1. Note that some crystals are older than others, and we have not observed any age-related affects to scintillator performance over a two-year timeframe, but a quantitative analysis of this claim is warranted and will be reported in a future publication. 
Table 1: LISe scintillator samples and measurement locations

\begin{tabular}{|c|c|c|c|c|c|c|}
\hline \multirow{2}{*}{\multicolumn{2}{|c|}{ Sample }} & \multirow[b]{2}{*}{ Boule } & \multirow{2}{*}{$\begin{array}{l}\text { Measurement } \\
\text { Location }\end{array}$} & \multirow{2}{*}{$\begin{array}{l}\text { Thickness } \\
\text { ( } \mu \mathrm{m})\end{array}$} & \multicolumn{2}{|c|}{ Surface roughness } \\
\hline & & & & & $\begin{array}{c}\mathrm{R}_{\mathrm{z}} \\
(\mu \mathrm{m})\end{array}$ & $\begin{array}{c}\mathrm{R}_{\mathrm{a}} \\
(\mu \mathrm{m})\end{array}$ \\
\hline \multicolumn{2}{|c|}{ LISe-1 } & 092613 & HFIR & 650 & $2.3 \pm 0.6$ & $0.085 \pm 0.014$ \\
\hline \multicolumn{2}{|c|}{ LISe-2 } & 092613 & PSI & 650 & -- & -- \\
\hline \multicolumn{2}{|c|}{ LISe-3 } & 010515 & HFIR/sCMOS & 520 & $1.69 \pm 0.15$ & $0.20 \pm 0.04$ \\
\hline \multirow{5}{*}{ LISe-4 } & Whole & \multirow{5}{*}{041714} & HFIR/sCMOS & 518 & -- & -- \\
\hline & A & & HFIR/CCD & 518 & $6.9 \pm 2.1$ & $0.19 \pm 0.03$ \\
\hline & $\mathrm{B}$ & & HFIR/CCD & 480 & $12.5 \pm 1.7$ & $0.39 \pm 0.04$ \\
\hline & $\mathrm{C}$ & & HFIR/CCD & 396 & $8.4 \pm 2.0$ & $0.461 \pm 0.014$ \\
\hline & $\mathrm{D}$ & & HFIR/CCD & 377 & $22.5 \pm 1.3$ & $0.806 \pm 0.018$ \\
\hline \multicolumn{2}{|c|}{ LISe-5 } & 041714 & HFIR/sCMOS & 452 & -- & -- \\
\hline \multirow{5}{*}{ LISe-6 } & Whole & \multirow{5}{*}{010515} & HFIR/sCMOS & 714 & -- & -- \\
\hline & 1 & & HFIR/CCD & 714 & $4.8 \pm 1.6$ & $0.6 \pm 0.4$ \\
\hline & 2 & & HFIR/CCD & 286 & $17 \pm 4$ & $0.4 \pm 0.3$ \\
\hline & 3 & & HFIR/CCD & 206 & $5.2 \pm 5$ & $0.19 \pm 0.03$ \\
\hline & 4 & & HFIR/CCD & 198 & $4.2 \pm 1.5$ & $0.16 \pm 0.05$ \\
\hline \multicolumn{2}{|c|}{ LISe-7 } & 010515 & HFIR/CCD & 960 & $0.9 \pm 0.4$ & $0.09 \pm 0.07$ \\
\hline
\end{tabular}

\subsection{Neutron Absorption Efficiency}

The neutron absorption efficiency, defined through equation 1, for each LISe sample is provided in Fig. 1. At the CG-1D beam line, the polychromatic neutron beam has a wavelength ranging from $0.8 \AA$ to $6 \AA$ with a peak at $2.6 \AA[12,19]$. For a stoichiometrically balanced LISe sample, the effective cold neutron wavelength at the CG-1D beamline was determined from the data to be $3.297 \AA$, and a continuous absorption efficiency curve for LISe, both total and ${ }^{6} \mathrm{Li}$, is also provided. From these results, it is clear that LISe has an intrinsic neutron absorption efficiency consistent with theory. For all samples, the standard deviation of absorption was only a few percent, indicating a very uniform lithium concentration. The deviation from a perfect fit may be due thickness variations across the sample, non-scintillating regions and inclusions, and imperfect stoichiometric ratio of the constituent elements due to the reactivity of lithium and selenium during growth. At this energy, a $50 \mu \mathrm{m} \mathrm{ZnS(Cu):}{ }^{6} \mathrm{LiF}(2: 1)$ scintillation screen with a $0.159 \mathrm{~g} / \mathrm{cm}^{3}{ }^{6} \mathrm{Li}$ potential for a much higher neutron utilization factor of the neutron imaging beam line, assuming each $\left.{ }^{6} \mathrm{Li}(\mathrm{n},)^{\mathrm{t}}\right)^{4} \mathrm{He}$ is registered by the detection system. 


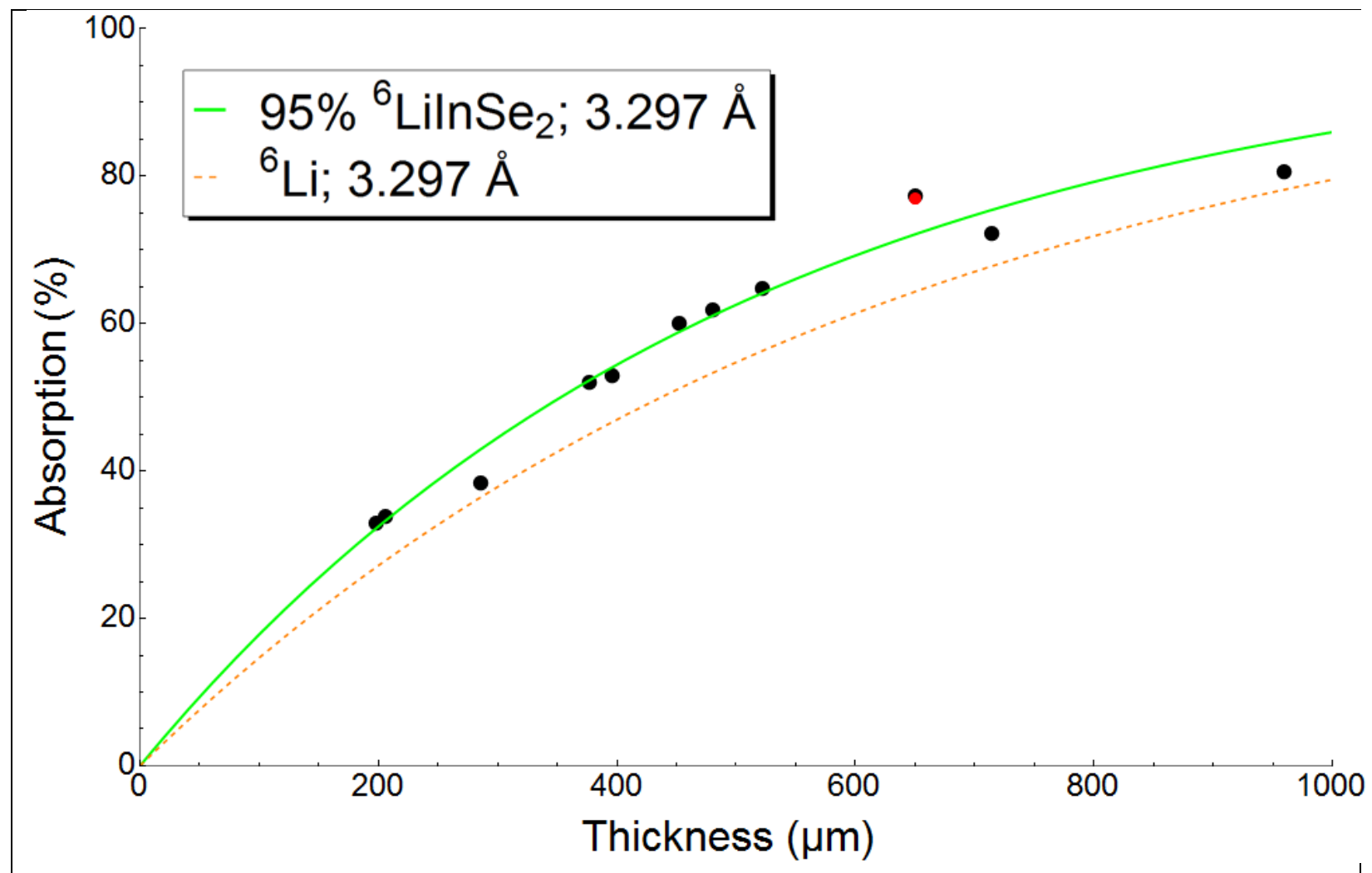

Fig. 1: Neutron absorption efficiency of LISe at various thicknesses. The red dot was measured on BOA at PSI [13] and the other samples on CG-1D at HFIR.

\subsection{Scintillation Relative Light Yield and Homogeneity}

The light intensity measured relative to the $50 \mu \mathrm{m} \mathrm{ZnS}(\mathrm{Cu}):{ }^{6} \mathrm{LiF}(2: 1)$ scintillation screen using the ANDOR ${ }^{\mathrm{TM}}$ CCD at HFIR and at PSI is provided in Fig. 2. The peak emission wavelength of LISe and the comparator scintillation screen differs by $18 \mathrm{~nm}$, which is also near the peak quantum efficiency of the ANDOR ${ }^{\mathrm{TM}}$ CCD used. Therefore, the direct comparison between the two provides a relatively accurate estimation of the relative light yield of each LISe sample. Further, in reference [11], the light yield of LISe is reported to be 9,000 photons/MeV, which was LISe-7 in this experiment. The light yield of LISe-7 could vary between this experiment and the experimental results reported in [11] due to surface oxidation, but does serve as a quantitative data point to estimate the true light yield of each LISe sample investigated within the constraints of the measurement technique in [11].

In Fig. 2, four different sets of data of RLY to $\mathrm{ZnS}(\mathrm{Cu}): \mathrm{LiF}$ are provided (see section 2); a large general set, a set for the four LISe-4 and LISe-6 pieces, and for LISe-2 measured at PSI. The RLY of all LISe samples of different thicknesses is between $1 \%$ and $20 \%$ with a standard deviation between $4-10 \%$ for all samples except LISe-6, which had a RLY variation of $22 \%$. The average RLY per micron found was $0.013 \pm 0.005$ with maximum and minimum values of 0.023 and 0.003 , respectively. The inverted radiograph of LISe-1 along with the measured light response from LISe1 is provided in Fig. 3. The percent spread in the neutron absorption of LISe- 1 is $3.4 \%$, which is considerably lower than its percent spread in the neutron-induced scintillation yield of 5.8\%. In Fig. 3, the open beam image shows spikes of light yield, where surface scratches are clearly visible. 


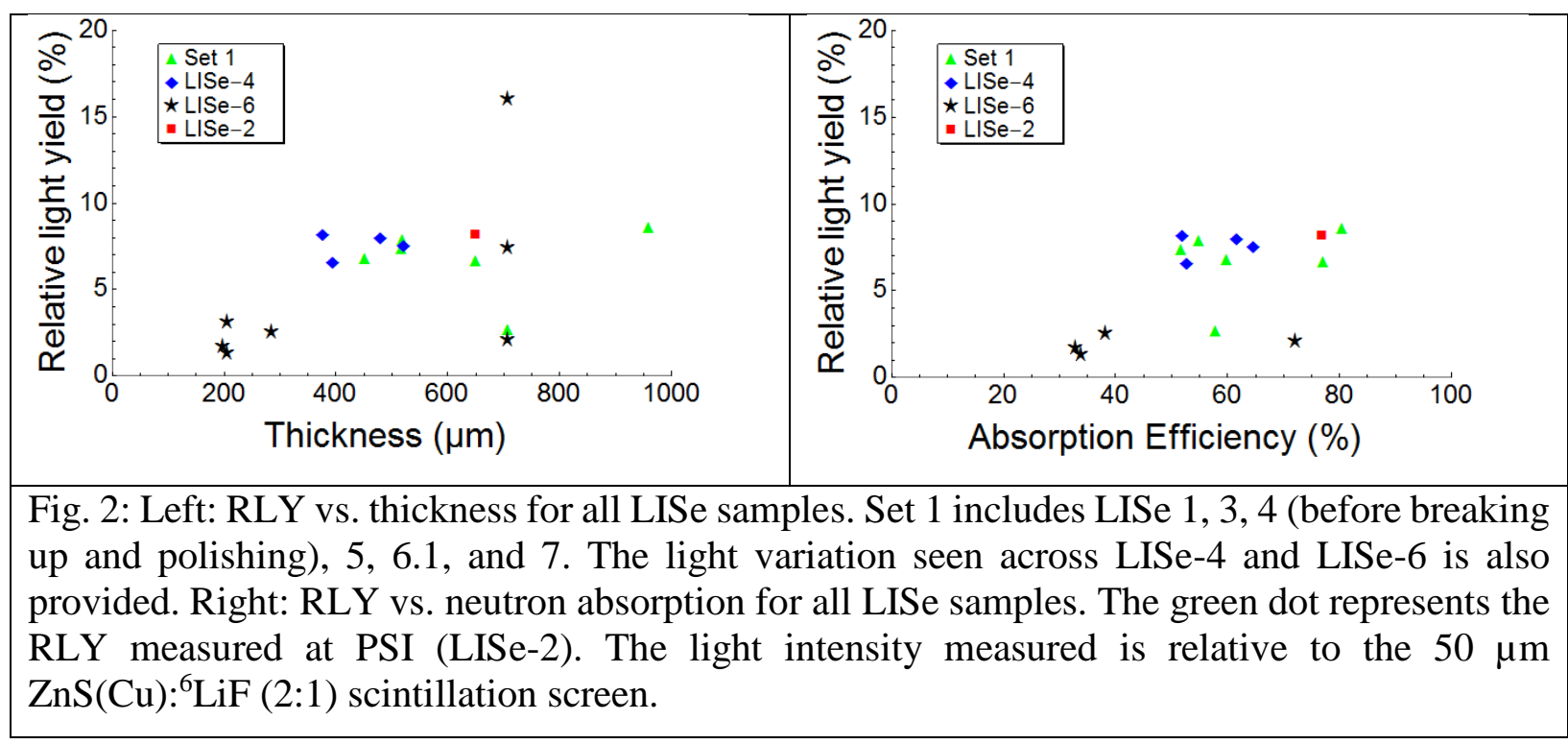

234

The variation of the RLY observed in Fig. 2 shows a general increasing trend with thickness, but there does exist a strong variability between data points. The strongest variation is for LISe-6.1 at $714 \mu \mathrm{m}$, where the RLY was measured at different spots on the crystal. Only LISe-6.1 and LISe6.3 (at $206 \mu \mathrm{m}$ ) displayed a varying and abrupt change in the RLY across the surface, discussed further in section 4.2.1.

Looking at the RLY as a function of absorption efficiency, there are two general groups. One of the grouped data points are for LISe-6 (the green triangle at $~ 700 \mu \mathrm{m}$ ) and LISe-6.1 through LISe6.4. These samples generally exhibited a lower RLY than other samples investigated, but the large range in RLY for the different sampled regions of LISe-6.1 is clearly present. The other group between 5-10\% RLY contains all the other samples. Therefore, upon inspection of Fig. 1 and 2, we would expect a larger change in the RLY than that observed. These results indicate that the RLY across the LISe samples does not strictly scale as a function of neutron absorption efficiency. This could be due to a variety of reasons, including variations in growth parameters affecting activation of the scintillation mechanism, alpha and triton secondary particle escape rate, and surface finish.

MCNPX v2.7 [20] simulations were conducted to estimate the rate of alpha and triton particle escape from the surface of LISe as a function of its thickness with a neutron energy of $3.297 \AA$. Of the secondary particles, the triton has the largest range of $40 \mu \mathrm{m}$ and dominates the escape rate over the alpha particle with a range of only $7 \mu \mathrm{m}$. The simulations tallied the quotient of the rates of full energy deposition to any energy deposition. It was found that the number of interactions that deposited the full Q-value of the reaction for a $200 \mu \mathrm{m}$ thick LISe sample was 88.3\%, versus 93.7\% for a $450 \mu \mathrm{m}$ thick LISe sample. The difference in the average energy deposition by the secondary particles for these thicknesses is $45.2 \%$. Correcting for absorption efficiency, the experimentally measured drop in RLY between LISe-5 and LISe-6.4 is $44.5 \%$. Comparing these two numbers, we can see that the RLY difference measured between LISe-5 and LISe-6.4 is wellexplained by particle escape. Still, upon further inspection of Fig. 2, it can be seen that the light yield is not increasing with the expected logarithmic-type behavior consistent with absorption 
efficiency for LISe sample thicknesses greater than $500 \mu \mathrm{m}$, which indicates that the light yield variation is more than just a function of absorption efficiency.

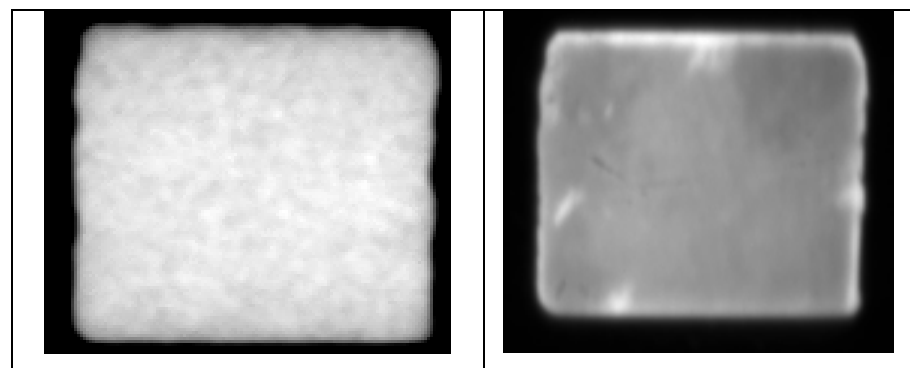

Fig. 3: Left: Absorption profile (inverted) of LISe1 , where the standard deviation of neutron absorption across the scintillator is $3.4 \%$. Right: Open beam image of LISe-1, where the measured light intensity varies across the sample by $5.8 \%$.

265

266

267

268

269

270

271

272

273

274

275

276

277

278

279

280

281

282

283

284

285

286
To further investigate this phenomenon, a study on the effect of surface finish on RLY was conducted using LISe-4, which was cut into four separate pieces and given different thicknesses and surface finishes. Referring to the surface roughness and thicknesses reported in Table 1 and the data provided in Fig. 2, it is clear that the RLY efficiency for LISe-4 increases with a rougher surface by $21 \%$. This is to be expected, since a rougher surface provides a random interface that increases the effective critical angle at which light can escape from the surface. The index of refraction of LISe is 2.5 [17], and this indicates that, for an air interface, the critical angle for complete internal reflection is $23.6^{\circ}$. The surface roughness increased the effective critical angle for the measured RLY to $28.5^{\circ}$. However, it should be noted that the RLY for LISe-4 samples A and C (smooth finish) and B and D (rough finish) yield similar RLY values for the same surface finish. Indeed, the light collection is a function of the location of interaction, because this dictates the solid angle of emitted light that will be collected, and it also affects the image sharpness measured by the CCD system.

As discussed by Williams et al. [9], the optical system must be optimized for the scintillation screen utilized. The tests performed here utilized a $\mathrm{ZnS}(\mathrm{Cu}) \cdot{ }^{6} \mathrm{LiF}$ optimized optical system, and therefore, the measured number of photons per neutron may be increased by adopting other optical systems, such as infinity-corrected optics. To fully understand the observed results, additional studies on the optical transfer systems is required. 


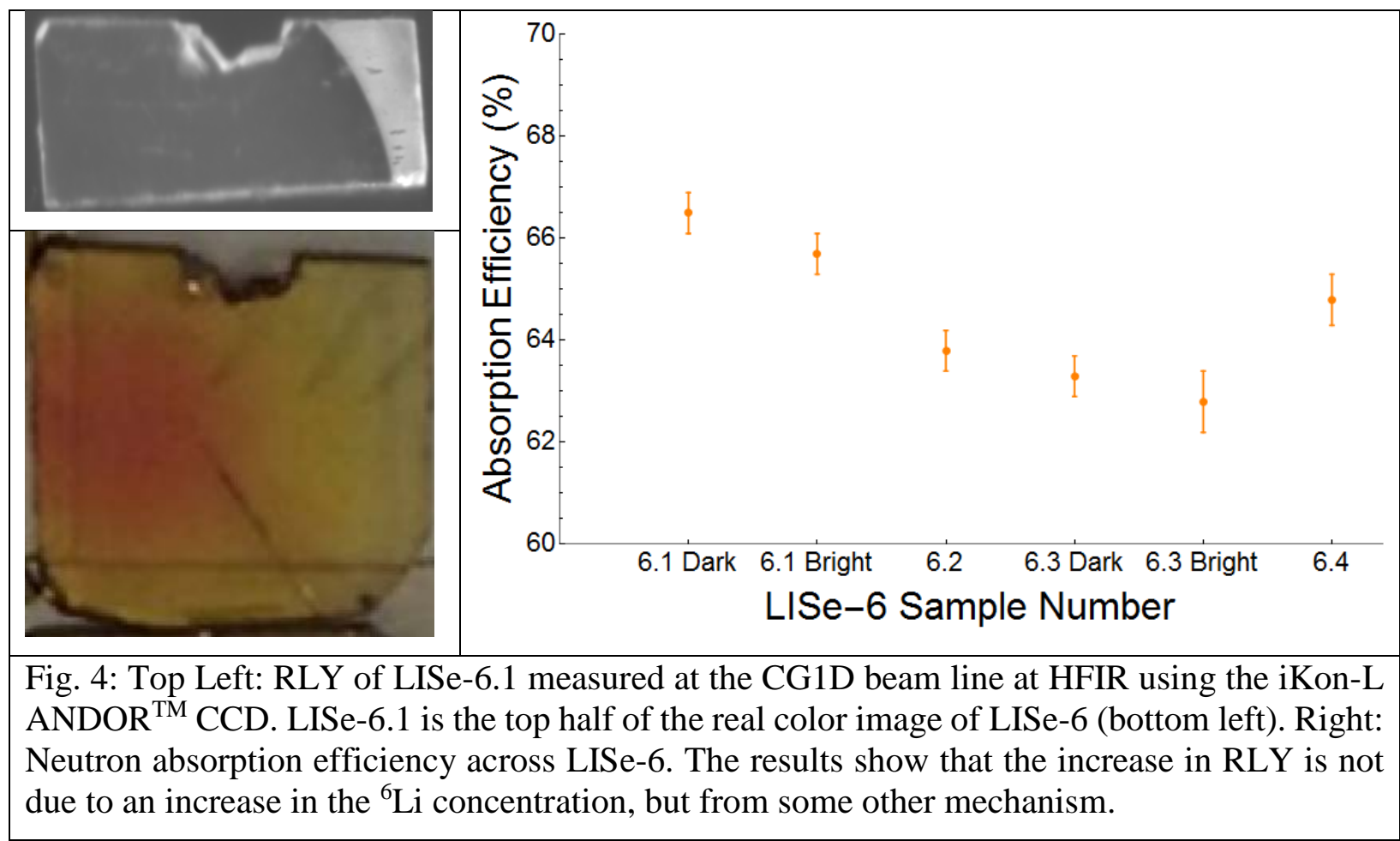

\subsubsection{Color Variations in LISe}

For sample LISe-6, a strong interface is present that shows a change in the RLY by a factor of eight. The RLY values for LISe-6.1 and LISe-6.3 is seen in Fig. 2 for the three black stars at 714 and two black stars at $206 \mu \mathrm{m}$, respectively. A comparison of a color image of LISe-6 and the open beam image of LISe-6.1 is provided in Fig. 4. Direct comparison indicates that the red and nearby yellow region correspond to a uniform, low RLY region, whereas the yellow-greenish color region exhibits a high RLY. The curvature of the interface suggests that the change in RLY is radially symmetric about the radius of the growth boule. Looking at the absorption of LISe-6 in Fig. 4 across the regions where LISe-6.1 through LISe-6.4 were cut from, the increase in RLY is not due to an increase in energy deposition rate/neutron utilization factor (i.e. the ${ }^{6} \mathrm{Li}$ concentration) within the crystal, but from the activation of the scintillation mechanism. The open beam image indicate a very sharp interface, and a knife-edge test of the sharp RLY interface resulted in an MTF at 10\% of $80 \mu \mathrm{m}$. Inspecting the real color image of LISe-6, the color transition does not appear to be this sharp (8.27 x $4.30 \mathrm{~mm}^{2}$ ), but displays a gradual change in color across a couple hundred microns. Therefore, the color of LISe is only a partial indicator of the scintillation light yield of LISe. Further investigation is underway of the relationship between crystal structure, impurities, apparent color, and scintillation efficiency of LISe.

\subsection{Exposure Memory and Radiation Hardness}

The potential memory effect of LISe due to the presence of ${ }^{115} \mathrm{In}$ was investigated on CG-1D at HFIR. ${ }^{115}$ In has a thermal neutron cross section $21.2 \%$ the value of ${ }^{6} \mathrm{Li}$, and the thermal neutron capture results in numerous high energy prompt gamma-rays ranging from $22 \mathrm{keV}$ to 6,656 keV. Further, the neutron capture results in the production of the ground and isomer states of ${ }^{116} \mathrm{In}$ with half-lives of 14.1 seconds and 54.29 minutes, respectively. The branching ratio of the isomer-to- 
ground state is 0.267 [21], and both decay via negatron emission. In addition, there exists a higher level state above ${ }^{116 \mathrm{~m}}$ In just described with a half-life of 2.18 seconds that decays via low energy gamma emission to the ${ }^{116 \mathrm{~m}}$ In state [22]. The presence of the radioactive ${ }^{116} \mathrm{In}$ capture products raises questions as to the viability of LISe for dynamic imaging studies because the decay of these isotopes may lead to residual scintillation.

To quantify the effect of ${ }^{115}$ In activation, a series of measurements were taken after exposing LISe1 to the CG-1D neutron beam for 49.7 minutes. For the first 200 seconds, images were captured with the CCD camera with ten-second integration times followed by sixty-second integration time images for an additional 30 minutes. Analysis of these images revealed that LISe showed no afterglow above the dark field, even in the first image. This advantageous result indicates that LISe is not subject to memory effects due to the exposure of neutrons at the flux intensity on CG-1D and the potential for dynamic imaging experiments. LISe's lack of neutron exposure memory is expected to be due to the low $\mathrm{Z}_{\text {eff }}$ of LISe, where the range of energetic betas and gamma-rays is very large, such that full energy deposition rarely occurs for beta particles above a few hundred keV.

Finally, LISe-1 was investigated three times on CG-1D at HFIR using the CCD camera. For two white field measurements separated by 668 minutes of exposure, the RLY increased by $9 \%,{ }^{1}$ which suggests that, either the neutron flux increased by a significant fraction between these two measurements, the light yield from the $\mathrm{ZnS}(\mathrm{Cu}):{ }^{6} \mathrm{LiF}$ scintillation screen from RC TRITEC decreased, or the light output of LISe-1 increases after being exposed to a neutron fluence of approximately $3 \times 10^{12} \mathrm{~cm}^{-2}$. It is known that the light yield of ZnS-based scintillation screens for neutron imaging is inversely related to exposure, where the largest change is observed during the first part of its useful life. The $\mathrm{ZnS}(\mathrm{Cu}):{ }^{6} \mathrm{LiF}$ screen used in these tests was installed on the CG1D neutron imaging beam at HFIR in December of 2014, so the expected reduction in light yield of this scintillation screen may be the reason for the apparent brightness change of LISe- 1 . The neutron damage created could also be a contributor for the measured relative light yield, since it is not beyond reason that the neutron-induced damage could enhance the scintillation mechanism.

\subsection{Spatial Resolution}

The spatial resolution measured at HFIR via the resolution parameter, $\alpha$, is provided in Fig. 5 . The full-width-at-half-maximum (FWHM) of the LSF is described by

$$
F W H M=\frac{2}{\alpha},
$$

which means that a larger $\alpha$ corresponds to a higher spatial resolution. Conducting a DFT on the averaged LSF, the spatial resolution for the LISe samples is provided at the $10 \%$ cutoff, also provided in Fig. 5. It can be observed that the spatial resolution measured by the sCMOS (LISe4 and LISe-6) and CCD (LISe-4 A-D and LISe-6.1) cameras were similar. The resolution measured for each LISe sample is relatively constant for thicknesses above $300 \mu \mathrm{m}$, indicating that the capable spatial resolution of LISe is not strictly a function of the thickness as is generally true for ZnS-based imagers. In reference to section 4.2.1, the knife-edge test was conducted across both the bright and dark regions of LISe-6.1. It was found that the bright region exhibited a $29 \%$ increase in the spatial resolution compared to the dark region. Investigating the averaged LSF of each, it was found that the averaged LSF was not smooth in the dark region, resulting in a faster

\footnotetext{
${ }^{1}$ The first 264 minutes of exposure was on December 12, 2014 (cycle 458), and the remaining 404 minutes of exposure was conducted on March 23, 2015 (cycle 459).
} 
decrease of the MTF (or a larger $\alpha$ ). However, if an assumed fit to the LSF in the dark region of LISe-6.1 is used, a near-identical resolution is obtained. Therefore, the difference seen in the spatial resolution between the bright and dark regions of LISe-6.1 is primarily due to statistical noise, or the RLY and total image acquisition time. Extending this argument to LISe samples above $300 \mu \mathrm{m}$, the same general agreement is found through inspection of Fig. 2. Below $300 \mu \mathrm{m}$, the spatial resolution of LISe increased rapidly, which does not follow the trend of RLY in Fig. 2. The reason for the observed increase in spatial resolution is unclear at this time, although one possible reason is that thinner samples exhibit more secondary particle surface escape and less internal light spreading before exiting the LISe sample for a given surface roughness via a lower optical path length. Finally, it was found that every LISe sample investigated produced a better resolution than the $50 \mu \mathrm{m} \mathrm{ZnS}(\mathrm{Cu}):{ }^{6} \mathrm{LiF}$. Therefore, although the $\mathrm{ZnS}(\mathrm{Cu}):{ }^{6} \mathrm{LiF}$ exhibits a much higher light yield, the relative resolution between $\mathrm{ZnS}(\mathrm{Cu}):{ }^{6} \mathrm{LiF}$ and $\mathrm{LISe}$ did not vary as a function of LISe thickness or relative light yield as one might expect.

At this point, it should be noted that the optical systems at HFIR were not modified in this experiment, but was at the assumed optimized setting for the $50 \mu \mathrm{m} \mathrm{ZnS}(\mathrm{Cu}){ }^{6} \mathrm{LiF}$ scintillation screen from RC TRITEC. The results obtained indicate that the combined focal length and depth of field of the optical systems at HFIR enabled the measured spatial resolutions for a variety of LISe scintillator thicknesses. It is likely the case that the optical clarity and high index of refraction of LISe contributed to the measured spatial resolution. However, the complete specifications of the optical systems (CCD and sCMOS) are proprietary, which did not allow a suitable computational investigation for direct comparison. Further study on the relationship between the optical transfer and digitation system design on spatial resolution of LISe is warranted.
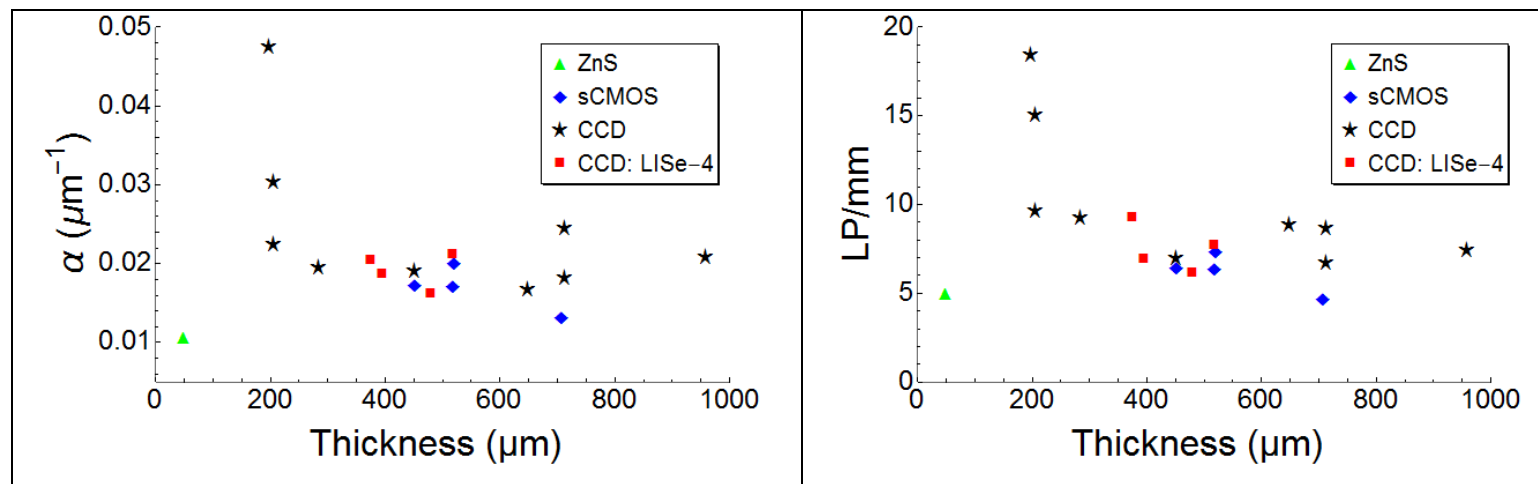

Fig. 5: Left: Resolution parameter, $\alpha$, versus LISe scintillator thickness. Right: $10 \%$ value of MTF for all LISe scintillators versus their thickness.

The effect of the beam divergence was quantified by conducting a knife-edge test on LISe-4D, LISe-5, and LISe-7 using the CCD camera system on CG-1D at HFIR for a separation distance between the knife-edge and front face of the scintillation crystals of 2.48 and $4.48 \mathrm{~cm}$. The beam aperture in this experiment was $16 \mathrm{~mm}$, corresponding to an $\mathrm{L} / \mathrm{D}$ ratio of 412 . The resolution parameter, $\alpha$, and the number of line pairs per millimeter for these three samples is provided in Table 2, where it can be seen that the resolution drops off, as expected. The relative change in the spatial resolution of these three samples is not equal. It can be seen that LISe-4D and 7 exhibit the largest change in the spatial resolution with a change in the object-to-LISe separation distance, but 
all samples approach the same resolution at the farther $4.48 \mathrm{~cm}$ separation distance. This result indicates that the beam divergence dominates the resolution measured by these three samples at a farther object-scintillator separation distance, but at smaller separations, other factors play a critical role in the measured spatial resolution, such as sample thickness, surface roughness, and RLY (signal-to-noise).

Table 2: Change in the measured system resolution as a function of knife-edge-to-LISe separation distance.

\begin{tabular}{c|c|c|c|c|c|c}
\hline \hline & \multicolumn{2}{|c|}{$2.48 \mathrm{~cm}$} & \multicolumn{2}{c|}{$4.48 \mathrm{~cm}$} & \multicolumn{2}{c}{$\Delta \mathrm{SR} / \mathrm{SR}_{0}$} \\
\hline Sample & $\begin{array}{c}\alpha_{0} \\
\left(\mu \mathrm{m}^{-1}\right)\end{array}$ & $(\mathrm{LP} / \mathrm{mm})_{\mathrm{o}}$ & $\begin{array}{c}\alpha_{1} \\
\left(\mu \mathrm{m}^{-1}\right)\end{array}$ & $(\mathrm{LP} / \mathrm{mm})_{1}$ & $\Delta \alpha$ & $\Delta \mathrm{LP} / \mathrm{mm}$ \\
\hline LISe-4D & 0.0203 & 9.2 & 0.0163 & 6.55 & 0.197 & 0.288 \\
\hline LISe-5 & 0.0190 & 6.96 & 0.0188 & 6.55 & 0.011 & 0.059 \\
\hline LISe-7 & 0.0207 & 7.37 & 0.0176 & 6.16 & 0.150 & 0.164 \\
\hline \hline
\end{tabular}

The MTF of the CCD camera, the neutron beam divergence, and samples LISe-1 and LISe-7 for an object-to-LISe separation distance of $2.48 \mathrm{~cm}$ is provided in Fig. 6. Through inspection, it can be seen that the CG-1D neutron imaging beam line at HFIR is not limited in its spatial resolution from beam divergence, but by the optical system and scintillator. LISe-1 exhibits the general trend of the sinc function "bounce" from the CCD (see equations 3 and 7). LISe-7 does not exhibit the same "bounce" in its MTF, possibly be due to its larger thickness compared to LISe-1, where the MTF is already at zero at this spatial frequency. The sCMOS, on the other hand, has an effective pixel pitch one-half that of the CCD, but the measured resolution of LISe-4 and LISe-6 was similar compared to measurements made with the CCD (LISe-4 A-D and LISe-6.1).

The knife-edge technique used allows the determination of the scintillator resolution beyond the Nyquist frequency (13.7 line pairs per millimeter) of the CCD, and the clear separation of the measured MTF from the beam divergence MTF implies that the thickness of the thicker LISe scintillators is the limiting factor in the overall system spatial resolution. For the thinner LISe samples, the resolution measured is near the CCD Nyquist frequency, which indicates that LISe has the potential to be a very high spatial resolution scintillator. Still, the low light output of LISe relative to the $50 \mu \mathrm{m} \mathrm{ZnS}(\mathrm{Cu}){ }^{6} \mathrm{LiF}$ RC TRITEC scintillation screen will require longer exposure times to attain the necessary signal-to-noise.

One question that remains after comparison of the RLY and resolution data begs the question as to whether scintillation light is generated volumetrically. As presented in Fig. 1, it is clear that the differential lithium concentration follows first order transport theory, and Fig. 2 indicates that the RLY of each scintillator varies greatly, not following the trend seen in Fig. 1. In Table 1, the samples investigated are from different boules, and this is not yet a commercial material. Comparing the RLY of LISe-6 and LISe-7, it becomes apparent that, within a single boule, the RLY varies by a factor of two or more. Therefore, in this experimental technique, it is hard to determine if the relatively constant spatial resolution measured is due to the optical clarity of LISe or due to an inhomogeneity of scintillation yield across the volume of the scintillator. 


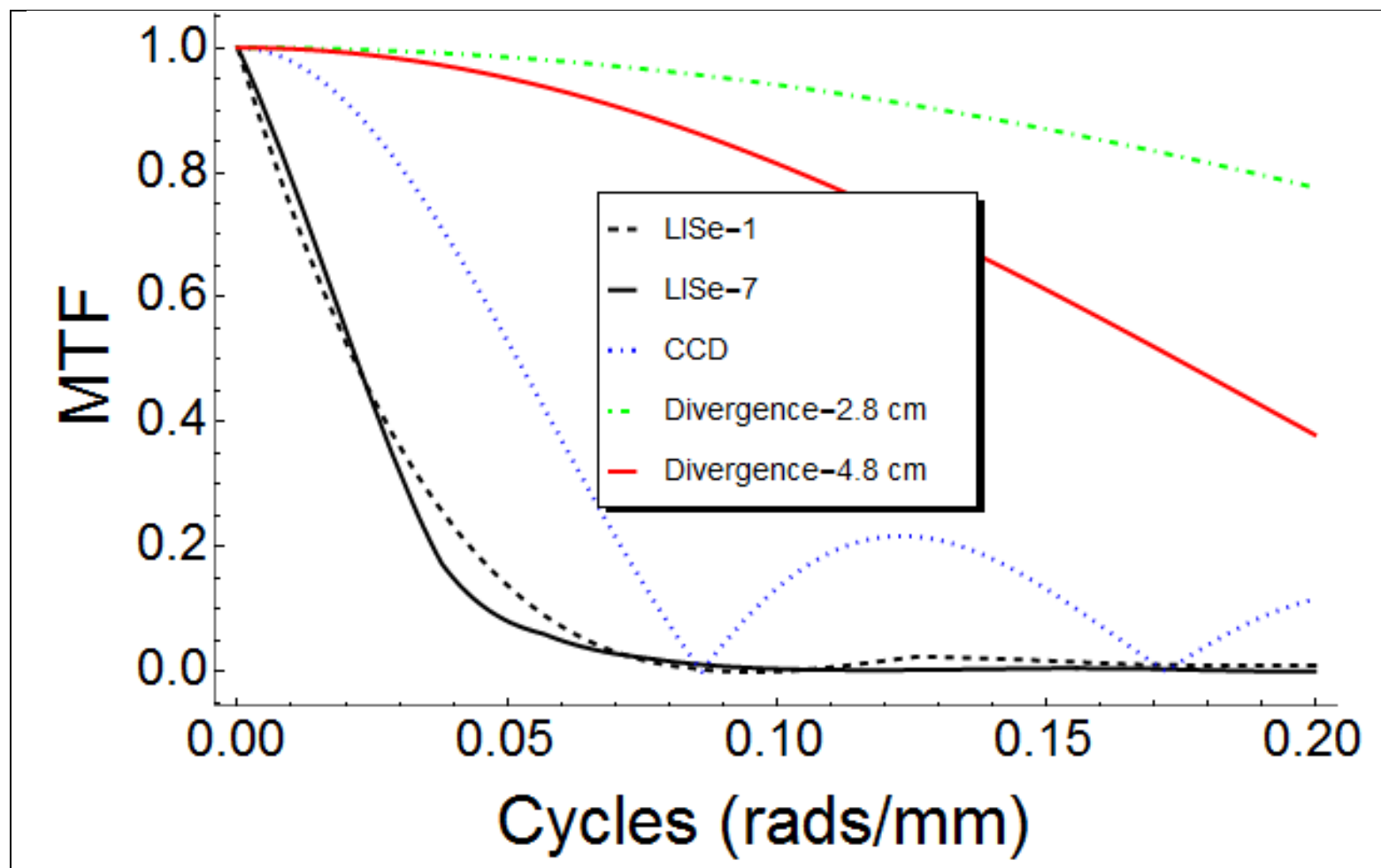

Fig. 6: MTF of LISe samples 1 and 7, the CCD camera, and the beam divergence for an L/D ratio of 412 for object-to-LISe separation distances of 2.8 and $4.8 \mathrm{~cm}$.

417 In an effort to address this possibility, we revisit the data presented for LISe-4 A-D and LISe-6.1 through 6.4. For LISe-4, it was found that the rougher surface yielded a higher RLY, which agrees with first principles. However, upon inspection of Fig. 5, the opposite trend is seen for the thinner LISe-4 crystals. For the thicker samples, the rougher surface, which increases the RLY, also increases the active area on the LISe crystal that allows light to be collected by the optical transfer system, thereby lowering the spatial resolution. For the thinner samples, however, the higher spatial resolution seen for LISe-4D is suspected to be due to a smaller light spread during transport to the crystal surface. Further, LISe-4D is the roughest sample, and also results in a larger light yield than the rough thicker sample LISe-4B with about one-half the surface roughness. Therefore, based upon the argument that a rougher surface yields higher light collection and lower spatial resolution, the results presented imply that the scintillation light generated per differential thickness follows first order transport theory.

For LISe-6-1, a significant change in RLY is seen across its face (see Fig. 4). The radial profile suggests that the change in light yield is more dependent in the radius of the boule rather than on the differential thickness. As already discussed, the abrupt change in RLY of LISe-6.1 is not yet understood. For the other LISe-6 samples, it is found that the spatial resolution increases significantly as the thickness is decreased by only a small amount. This suggests that the transport of the scintillation light within the bulk to the surface of LISe contributes considerably to the measured resolution. Further, the differential scintillation light generated at a given depth in all LISe samples, assuming it is homogeneous across its volume, is largest at its front face, following first order transport theory. This means that light transport properties are preferentially described 
at the back face of the crystal with respect the optical transfer system. Therefore, when the scintillator is very thin, the resolution increases by reducing the mean optical path length of the light traveling through the LISe scintillator.

These results do not disprove the possibility that the light is generated only in a differential volume of the LISe, but the current results suggest otherwise. The various factors in collected light, including scintillation efficiency, bulk scattering, interface scattering at each face, and surface roughness all play a role. The authors of this paper believe that the variability in RLY and spatial resolution is due to variations in the activation of the scintillation mechanism, crystal inclusions, and optical transport effects. The results presented do, however, indicate that further investigation is required to fully characterize the observed properties of LISe for cold neutron imaging.

\subsection{Image Reconstruction}

Beyond the knife-edge test, LISe-1 and LISe-2 were tested for their ability to reconstruct an image. Two objects were investigated, the PSI Siemens Star [15] and a 3D printed Tennessee Power T. LISe-1 was tested for both objects on CG-1D at HFIR, and LISe-2 was tested only with the Siemens Star on the BOA at PSI [13].

\subsubsection{Imaging Tests on BOA at PSI}

Eleven images were taken of the Siemens Star with ten second integration times. The eleven images were summed together to increase signal-to-noise, followed by a noise reduction filter. The resulting image is provided in Fig. 7, where the inner spoke seen corresponds to the $200 \mu \mathrm{m}$ line. Close inspection of this image indicates that the resolution is lower than this, but due to the sample size, a full image of the Siemens Star could not be conducted. Further, there are several features visible within the image not part of the Siemens Star, and they are a result of a combination of hot pixels (high energy background radiation interacting within the CCD) and surface features affecting light transport to the CCD via the optical coupling system. The observed features are not due to clusters of ${ }^{6} \mathrm{Li}$ atoms, or spikes in the neutron absorption efficiency.

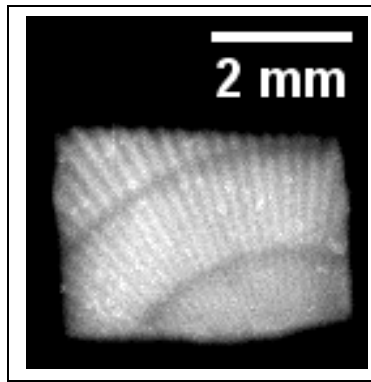

Fig. 7: Resolution measurement of LISe-2 using the PSI Siemens Star [15] at the BOA beam line [13].

\subsubsection{Imaging Tests on CG-1D at HFIR}



minimal artifacts from the stitching process.

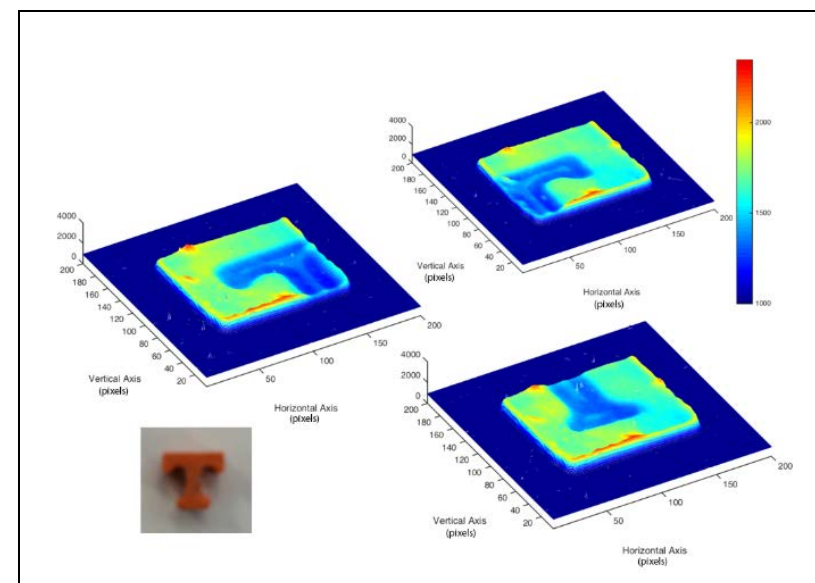

Larger area images were generated by translating the object around the active area of LISe-1. Each image was corrected for the dark and flat field. Generated images at each position were cropped to remove potential converter edge effects and then combined to create the composite image. For 3D printed University of Tennessee Power T, as shown in Fig. 8, three image positions were used to generate the composite image. It can be seen that the method used is suitable in generating images larger than a single crystal of LISe, where the direct stitching of the three images produces minimal artifacts at image interfaces. The image generated accurately displays the internal features of the Power $\mathrm{T}$, and when compared to the image generated by the $50 \mu \mathrm{m} \mathrm{ZnS}(\mathrm{Cu}) \cdot{ }^{6} \mathrm{LiF} \mathrm{RC}$ TRITEC scintillation screen (not shown), the same features are present.

The PSI Siemens Star [15] was imaged by taking 60 images over 12 spatial locations, all with 60 second integration times. The images were stitched together to create one quadrant of the Siemens Star. A higher contrast was obtained at the center of the Siemens Star by taking five images with a 300 second integration time for each. These two regions are defined by the red wedge and yellow box (center region) shown in Fig. 9. The resulting composite image of the Siemens Star shows

The composite image generated with LISe-1 was overlain on an image generated by the RC TRITEC $50 \mu \mathrm{m} \mathrm{ZnS(Cu):}{ }^{6} \mathrm{LiF}$ scintillation screen for direct visual comparison (acquisition parameters given in the blue box on the top left of Fig. 9). Through visual inspection, the image resolution of LISe- 1 is equivalent to the $50 \mu \mathrm{m} \mathrm{ZnS}(\mathrm{Cu}){ }^{6} \mathrm{LiF}$ RC TRITEC scintillation screen for the same acquisition parameters and generally agrees with the images taken on BOA at PSI (see Fig. 7). This indicates that LISe is capable of achieving a resolution equal to that of a thin ZnSbased converter screen when it is many times thicker than the achievable spatial resolution. The MTF study of spatial resolution indicated a superior resolution of LISe over a $50 \mu \mathrm{m} \mathrm{ZnS(Cu):}{ }^{6} \mathrm{LiF}$ scintillation screen, and the discrepancy is attributed to the low RLY. Indeed, for the central imaged region, LISe-1 shows a somewhat superior spatial resolution over the $50 \mu \mathrm{m} \mathrm{ZnS}(\mathrm{Cu}):{ }^{6} \mathrm{LiF}$ scintillation screen. Therefore, as LISe is further developed and appropriate optical systems are utilized, LISe may prove a better scintillator for cold neutron imaging applications.

Fig. 8: Left: Individual images taken of portions of a University of Tennessee "Power T" and a real color image of the object. Right: Reconstructed full image of the Power T. 


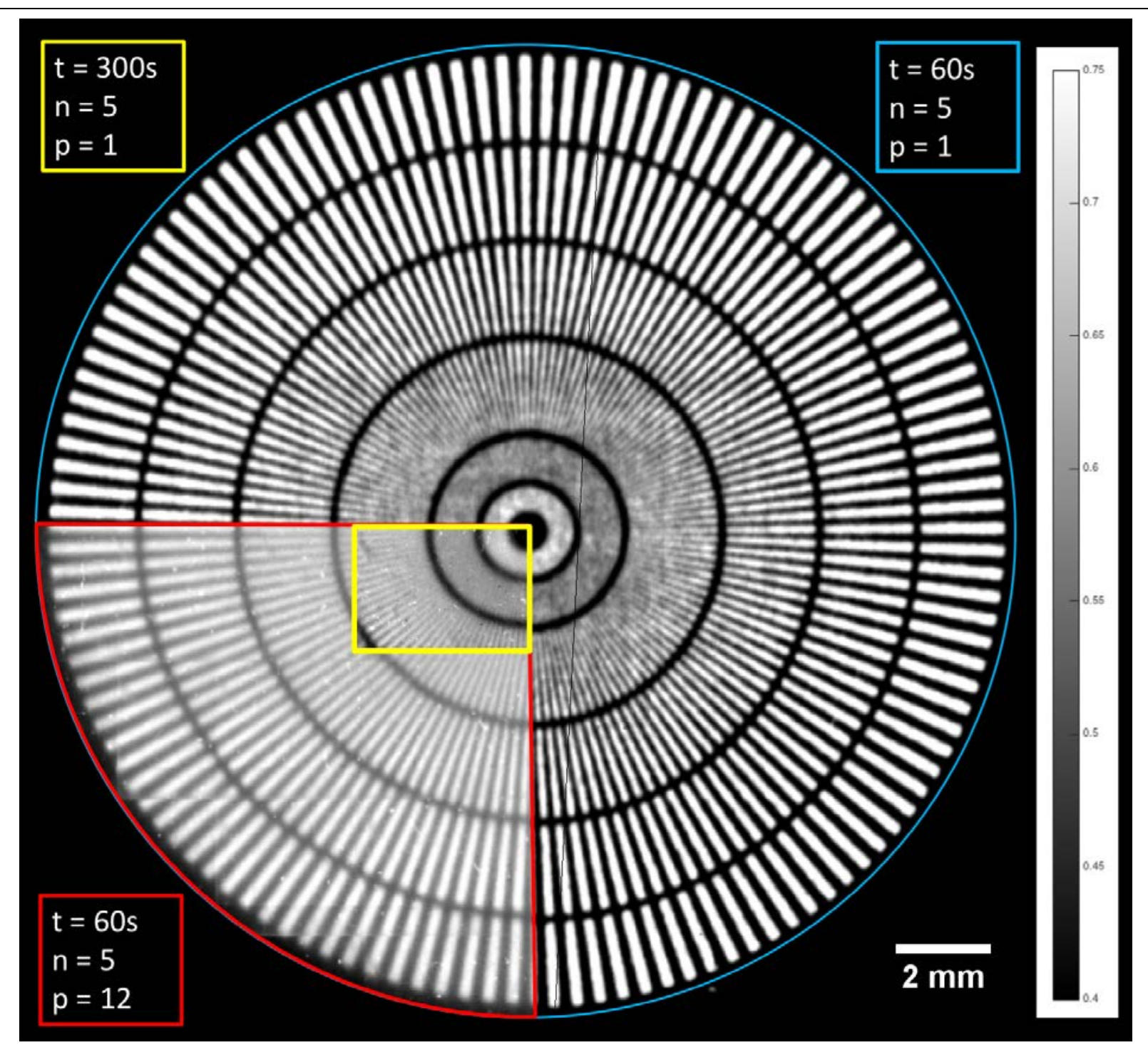

Fig. 9: Radiograph taken of the PSI Siemens Star [15] with the $50 \mu \mathrm{m} \mathrm{ZnS(Cu):}{ }^{6 i F} \mathrm{RC}$ TRITEC scintillation screen. Overlaid on the radiograph is data taken with LISe-1. In the colored regions, the integration time $(\mathrm{t})$, number of images taken to define the median image (n), and the number of positions of LISe used to define the composite image (p) is provided.

\section{Conclusion}

LISe is a new scintillation material that is highly sensitive to slow neutrons. The isotopic enrichment of ${ }^{6} \mathrm{Li}$ yields a very high neutron utilization factor. The well-behaved neutron absorption as a function of thickness suggests that the ${ }^{6} \mathrm{Li}$ is uniformly distributed through its bulk and not concentrated on the surface. LISe was found to exceed the capable spatial resolution of a $50 \mu \mathrm{m}$ thick $\mathrm{ZnS}(\mathrm{Cu}):^{6} \mathrm{LiF}$ from RC TRITEC, by as much as a factor of three for the thinner LISe samples investigated, even though all were considerably thicker than $50 \mu \mathrm{m}$. However, the low RLY of LISe compared to the $50 \mu \mathrm{m}$ thick $\mathrm{ZnS}(\mathrm{Cu}):{ }^{6} \mathrm{LiF}$ from RC TRITEC resulted in higher 
signal-to-noise for an equivalent measurement time. Further, the null neutron exposure memory result from the presence of ${ }^{115}$ In in LISe indicates that LISe could be used for transient neutron imaging studies (e.g., water flow in a PEM fuel cell).

The numerous RLY values reported for LISe clearly indicates that its optimization for cold neutron imaging has yet to be realized. For instance, rougher LISe surfaces lowered the measured spatial resolution for thicker samples but increased the spatial resolution for thinner samples. The likely mechanisms behind the observed result is a combination of the effective critical angle dictating internal reflection and the mean optical path length of scintillation photons. Further, the results presented for LISe-6 indicate that there is the potential for a significant increase in the RLY of LISe. Additional studies are needed to realize the potential of LISe for high resolution cold neutron imaging, including a more in-depth study of surface finish and scintillator thickness on spatial resolution and RLY, optimization of the optical transfer system, and effective activation of the scintillation mechanism.

\section{Acknowledgements}

This work is supported through subcontract number 4300090406 from CNS Y-12 National Security Complex and this material is based upon work supported under a Department of Energy Nuclear Energy University Programs Graduate Fellowship. A portion of this research at ORNL's High Flux Isotope Reactor was sponsored by the Scientific User Facilities Division, Office of Basic Energy Sciences, U.S. Department of Energy. Part of this work is based on experiments performed at the Swiss spallation neutron source SINQ, Paul Scherrer Institut, Villigen PSI, Switzerland.

\section{7. $\quad$ References}

1. Kardjilov, N., Fiori, F., Giunta, G., Hilger, A., Rustichelli, F., Strobl, M., Banhart, J., and Triolo, R., Neutron tomography for archaeological investigations. Journal of Neutron Research, 2006. 14(1): p. 29-36.

2. $\quad$ Strobl, M., Manke, I., Kardjilov, N., Hilger, A., Dawson, M., and Banhart, J., Advances in neutron radiography and tomography. Journal of Physics D: Applied Physics, 2009. 42(24): p. 243001.

3. Tobin, K.W., Bingham, P.R., and Gregor, J., Mathematics of Neutron Imaging, in Neutron Imaging and Applications, I.S. Anderson, R.L. McGreevy, and H.Z. Bilheux, Editors. 2009, Springer Science Business Media. p. 110-114.

4. Sakata, I., Ueda, T., Murakawa, H., Sugimoto, K., Asano, H., Takenaka, N., Yasuda, R., Tomura, T., and Shiozawa, M., Three-dimensional observation of water distribution in PEFC by neutron CT. Nuclear Instruments and Methods in Physics Research A, 2009. 605: p. 131-133.

5. $\quad$ Crow, L., Neutron Detectors for Imaging, in Neutron Imaging and Applications, I.S. Anderson, R.L. McGreevy, and H.Z. Bilheux, Editors. 2009, Springer Science Business Media. p. 47-49.

6. Trtik, P. and Lehmann, E.H., Isotopically-enriched gadolinium-157 oxysulfide scintillator screens for the high-resolution neutron imaging. Nuclear Instruments and Methods in Physics Research Section A: Accelerators, Spectrometers, Detectors and Associated Equipment, 2015. 788: p. 67-70.

7. Trtik, P., Hovind, J., Grünzweig, C., Bollhalder, A., Thominet, V., David, C., Kaestner, A., and Lehmann, E.H., Improving the Spatial Resolution of Neutron Imaging at Paul 
Scherrer Institut - The Neutron Microscope Project. Physics Procedia, 2015. 69: p. 169176.

8. Trtik, P. and Lehmann, W.H., Progress in High-resolution Neutron Imaging at the Paul Scherrer Institut - The Neutron Microscope Project. Journal of Physics, 2016.

9. Williams, S.H., Hilger, A., Kardjilov, N., Manke, I., Strobl, M., Douissard, P.A., Martin, T., Riesemeier, H., and Banhart, J., Detection system for microimaging with neutrons. Journal of Instrumentation, 2012. 7(02): p. P02014.

10. Stowe, A.C., Burger, A., and Groza, M., Bulk semiconducting scintillator device for radiation detection 2014: United States of America.

11. Wiggins, B., Groza, M., Tupitsyn, E., Lukosi, E., Stassun, K., Burger, A., and Stowe, A., Scintillation properties of semiconducting ${ }^{6}$ LiInSe $_{2}$ crystals to ionizing radiation Nuclear Instruments and Methods in Physics Research A, 2015. 801: p. 73-77.

12. Crow, L., Robertson, L., Bilheux, H., Fleenor, M., Iverson, E., Tong, X., Stoica, D., and Lee, W.T., The CG1 instrument development test station at the high flux isotope reactor. Nuclear Instruments and Methods in Physics Research Section A: Accelerators, Spectrometers, Detectors and Associated Equipment, 2011. 634(1, Supplement): p. S71S74.

13. Morgano, M., Peetermans, S., Lehmann, E.H., Panzner, T., and Filges, U., Neutron imaging options at the BOA beamline at Paul Scherrer Institut. Nuclear Instruments and Methods in Physics Research Section A: Accelerators, Spectrometers, Detectors and Associated Equipment, 2014. 754(0): p. 46-56.

14. Harms, A.A. and Wyman, D.R., Mathematics and physics of neutron radiography. Vol. 1. 1986: Springer Science \& Business Media.

15. Grunzweig, C., Frei, G., Lehmann, E., Kuhne, G., and David, C., Highly absorbing gadolinium test device to characterize the performance of neutron imaging detector systems. Review of Scientific Instruments, 2007. 78(5): p. 053708-053708-4.

16. Anderson, I.S., McGreevy, R.L., and Bilheux, H.Z., Neutron imaging and applications. Springer Science Business Media, 2009. 200: p. 987-0.

17. Nikogosyan, D.N., Nonlinear optical crystals: a complete survey. 2006: Springer Science \& Business Media.

18. Tobin, K.W., Brenizer, J.S., and Mait, J.N., Modulation transfer function technique for real time radioscopic system characterization. Applied Optics, 1989. 28(23): p. 50025009.

19. Kang, M., Bilheux, H.Z., Voisin, S., Cheng, C.L., Perfect, E., Horita, J., and Warren, J.M., Water calibration measurements for neutron radiography: Application to water content quantification in porous media. Nuclear Instruments and Methods in Physics Research Section A: Accelerators, Spectrometers, Detectors and Associated Equipment, 2013. 708(0): p. 24-31.

20. Pelowitz, D.B., MCNPX User's Manual. 2011: Los Alamos National Laboratory.

21. Beckurts, K.H., Brose, M., Knoche, M., Kruger, G., Pönitz, W., and Schmidt, H., Thermal Activation Cross Sections and Resonance Integrals of In 115. Nuclear Science and Engineering, 1963. 17(3): p. 329-335.

22. $\quad$ Blachot, J., Nuclear Data Sheets for A = 116. Nuclear Data Sheets, 2010. 111(3): p. 717895. 